$05,11,12$

\title{
Исследование моделей магнитных дендримеров методом Ванга-Ландау
}

\author{
(C) М.А. Магомедов ${ }^{1,2}$, М.М. Исаева ${ }^{1}$ \\ ${ }^{1}$ Институт фризики им. Х.И. Амирханова ДФИЦ РАН, \\ Махачкала, Россия \\ ${ }^{2}$ Отдел математики и информатики ДФИЦ РАН, \\ Махачкала, Россия \\ E-mail: magomedov_ma@mail.ru
}

Поступила в Редакцию 30 декабря 2019 г.

В окончательной редакции 30 декабря 2019 г.

Принята к публикации 10 января 2020 г.

Методом Монте-Карло изучены термодинамические свойства моделей магнитных дендримеров. Рассчитана плотность состояний системы и определены структуры основного состояния. Вычислены температурные зависимости намагниченности $m$, энтропии $S$, внутренней энергии $E$ и теплоемкости $C$. Показано, что в исследованной модели дендримера влияние поверхностных узлов на общую картину поведения системы с ростом размеров системы не ослабевает.

Ключевые слова: дендримеры, магнетизм, фазовые переходы, структура основного состояния, плотность состояний, алгоритм Ванга-Ландау, метод Монте-Карло.

DOI: $10.21883 /$ FTT.2020.05.49233.18M

\section{1. Введение}

В последние годы ведутся активные исследования как теоретическими, так и экспериментальными методами различных наноструктур [1-7]. Значительный интерес к таким структурам обусловлен рядом уникальных свойств и перспективой их широкого применения в различных областях науки и техники. Одним из таких перспективных направлений является адресная доставка лекарственных сред (АДЛ). Суть АДЛ заключается в том, что само лекарственное средство, а чаще средство его доставки (наноконтейнер с лекарством) доставляется непосредственно в заданную область органа или клетки. В результате лекарственное средство попадает точно в цель, не затрагивая другие органы. Несмотря на большие перспективы АДЛ сталкивается с значительными трудностями. Кратко задачу АДЛ можно сформулировать следующим образом: требуется разработать наноконтейнер для лекарства, которое само не вступает в химические реакции, каким-либо образом способствует транспорту лекарства в требуемое место в организме и позволяет произвести контролируемое высвобождение лекарства в нужный момент времени. Для адресной доставки лекарств разработано множество различных наноструктур: липосомы, мицеллы, хитозан, магнетит, нанотрубки, дендримеры, металлические наночастицы, суперпарамагнитные частицы пористый кремний и т.д. В последние годы для АДЛ стали широко применять магнитные наночастицы. Магнитные наночастицы можно направлять, прикладывая внешнее магнитное поле. Также прикладывая сильные переменные магнитные поля в заданной области можно провести нагрев и или контролируемое разрушение контейнера с выделением лекарства. Широкое применение при АДЛ получили высокомолекулярные соединения с сильно разветвленной древовидной структурой, получившие название ,дендримеры“. Отличительной особенностью дендритных структур от других нанообъектов считается возможность контролировать свойства и строение на всех этапах роста. Таким образом, высокая точность предсказания характеристик и размеров макромолекулы делают перспективным его применение в медицине в качестве агентов для АДЛ. К настоящему времени уже показана высокая эффективность дендримеров при доставке ряда лекарственных препаратов в экспериментах на животных. Несмотря на достигнутые успехи, на сегодняшний день все еще остаются открытыми вопросы, касающиеся эффективности и безопасности применения различных наноструктур для АДЛ [2-4]. Физические свойства таких систем изучены слабо. В связи с этим, в данной работе нами проведены исследования термодинамических характеристик моделей магнитных дендримеров.

\section{2. Модель и метод исследования}

Структура дендримера представляет собой ядро, ветви и концевые группы. Рост дендримера осуществляется, во всех направлениях, таким образом, дендритная структура представляет собой сферические слои, равноудаленные от центра. Ядро дендримера является нулевой точкой ветвления (его еще называют нулевой генерацией). Внешний слой заканчивается поверхностными, или терминальными, функциональными 
группами. Число терминальных групп возрастает в геометрической прогрессии при увеличении генерации, и ограничено, в свою очередь, площадью поверхности дендримера, которая имеет квадратичную зависимость от радиуса. Большинство наноразмерных дендримеров являются аморфными веществами и обладают эластичностью, также они обладают способностью набухать, изменять свои размеры. Физико-химические свойства дендритных структур во многом определяют концевые группы, которые лежат в основе классификации дендримеров [7].

На рис. 1 представлена модель магнитного дендриме$\mathrm{pa}$, исследованная в данной работе. Показано ядро дендримера, состоящее из трех узлов, а также узлы вплоть до четвертой генерации. Как видно из рисунка, число узлов в $i$-ом слое равно $N_{i}=3 \cdot 2^{i}$, таким образом, в каждом новом слое два раза больше узлов, чем в предыдущем. Общее число узлов в дендримере размера $R$ можно определить по формуле

$$
N=3+6+12 \ldots N_{r}=3 \sum_{i=0}^{R} 2^{i}=3 \cdot\left(2^{R+1}-1\right) .
$$

При четном числе генераций $R$ число узлов в нечетных слоях составляет $N_{1}=2 \cdot\left(4^{R / 2+1}-1\right)$, а в четных слоях $N_{2}=3+4 \cdot\left(4^{R / 2+1}-1\right)$. Таким образом, при больших $R$ мы имеем $N_{2} / N_{1} \rightarrow 2$, следовательно, треть узлов будут находиться в нечетных слоях, а две трети в четных.

Нами учитывалось обменное взаимодействие между узлами в ядре, между узлами ближайших генераций, a также между ближайшими парами одной генерации. Спины в узлах решетки могут принимать два значения $S= \pm 1$. Таким образом, гамильтониан модели дендримера может быть записан в следующем виде:

$$
H=-J_{0} \sum_{\langle i, j\rangle} S_{i} S_{j}-J_{1} \sum_{\langle i, j\rangle} S_{i} S_{j}-J_{2} \sum_{\langle i, j\rangle} S_{i} S_{j},
$$

где обменное взаимодействие в ядре учитывает первая сумма, вторая сумма учитывает взаимодействие вдоль ветви дендритной структуры, а третья сумма учитывает взаимодействия между соседними узлами. В данной работе нами полагалось $J_{0}=J_{2}$.

Наиболее эффективным инструментом моделирования наноструктур является метод Монте-Карло (МК) [8-18]. Одним из наиболее эффективных вариантов метода МК является алгоритм Ванга-Ландау [8-12]. Данный алгоритм является реализацией метода энтропийного моделирования и позволяет рассчитать функцию плотности состояний системы.

Алгоритм Ванга-Ландау в настоящей работе был использован в следующем виде [8-11].

Задается произвольная начальная конфигурация спинов. Стартовые значения плотности состояний $g(E)=1$, гистограммы распределений по энергиям $H(E)=0$, стартовый модификационный фактор $f=f_{0}=e^{1}$ $\approx 2.71828$. Многократно совершаем шаги в фазовом

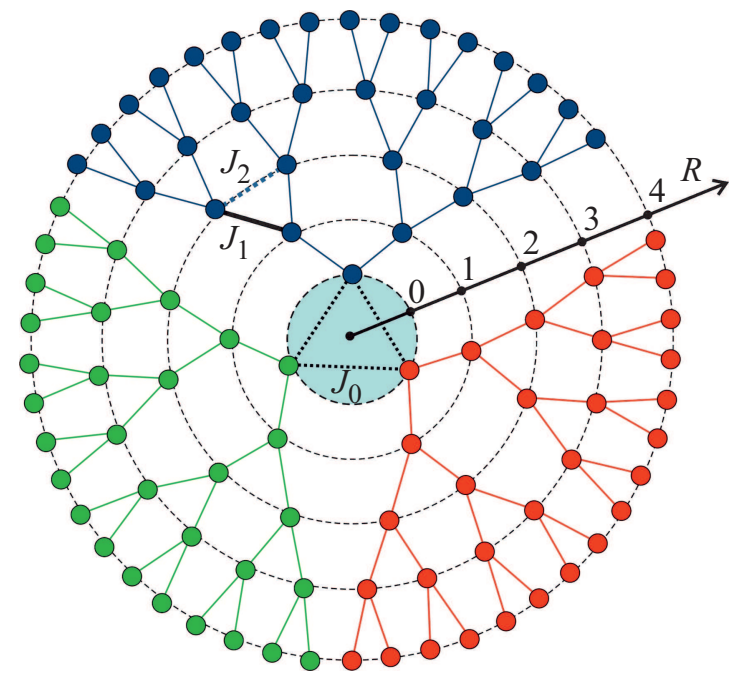

Рис. 1. Модель магнитного дендримера.

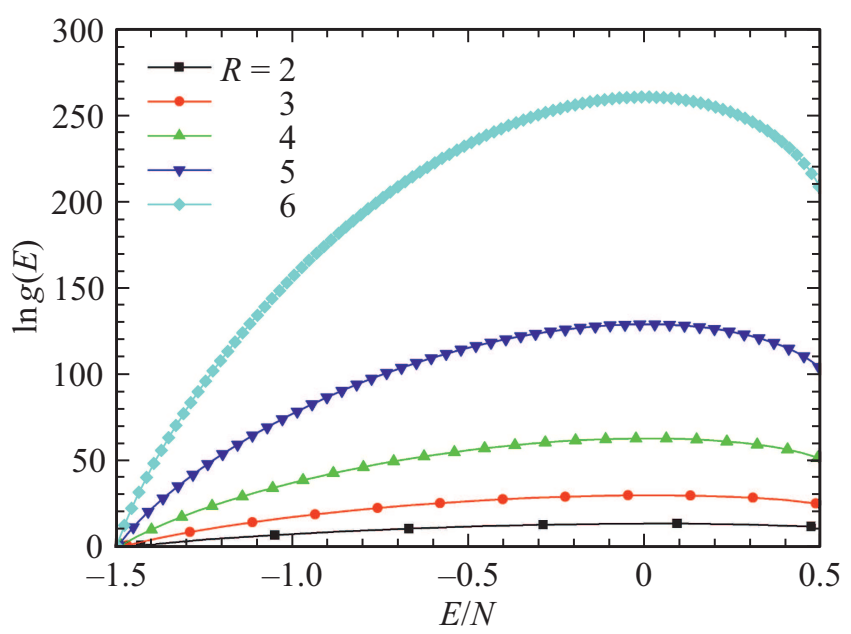

Рис. 2. Плотность состояний $g(E)$ для систем с размерами $R$ при $J_{1}=-1$ и $J_{2}=1$.

пространстве, пока не получим относительно плоскую гистограмму $H(E)$ (т.е. пока не будут посещены примерно одинаковое количество раз все возможные энергетические состояния системы). При этом вероятность перехода из состояния с энергией $E_{1}$ в состояние с энергией $E_{2}$ определяется по формуле $p=g\left(E_{1}\right) / g\left(E_{2}\right)$. Если переход в состояние с энергией $E_{2}$ состоялся, то $g\left(E_{2}\right)$ присваивается значение $f \times g\left(E_{2}\right)$, а гистограмма посещений $H\left(E_{2}\right)$ увеличивается на единицу, иначе $g\left(E_{1}\right)$ присваивается значение $f \times g\left(E_{1}\right)$ и $H\left(E_{1}\right)$ увеличивается на единицу. Если гистограмма стала „Плоской“ то обнуляем гистограмму $H(E) \rightarrow 0$, уменьшаем модификационный фактор $f \rightarrow \sqrt{f}$, и продолжаем снова, пока $f \geq f_{\max }$. В нашем случае $f_{\max }=1.0000000001$.

В стандартную методику алгоритма нами добавлена процедура, которая позволяет определить магнитную структуру основного состояния системы. Каждый раз 

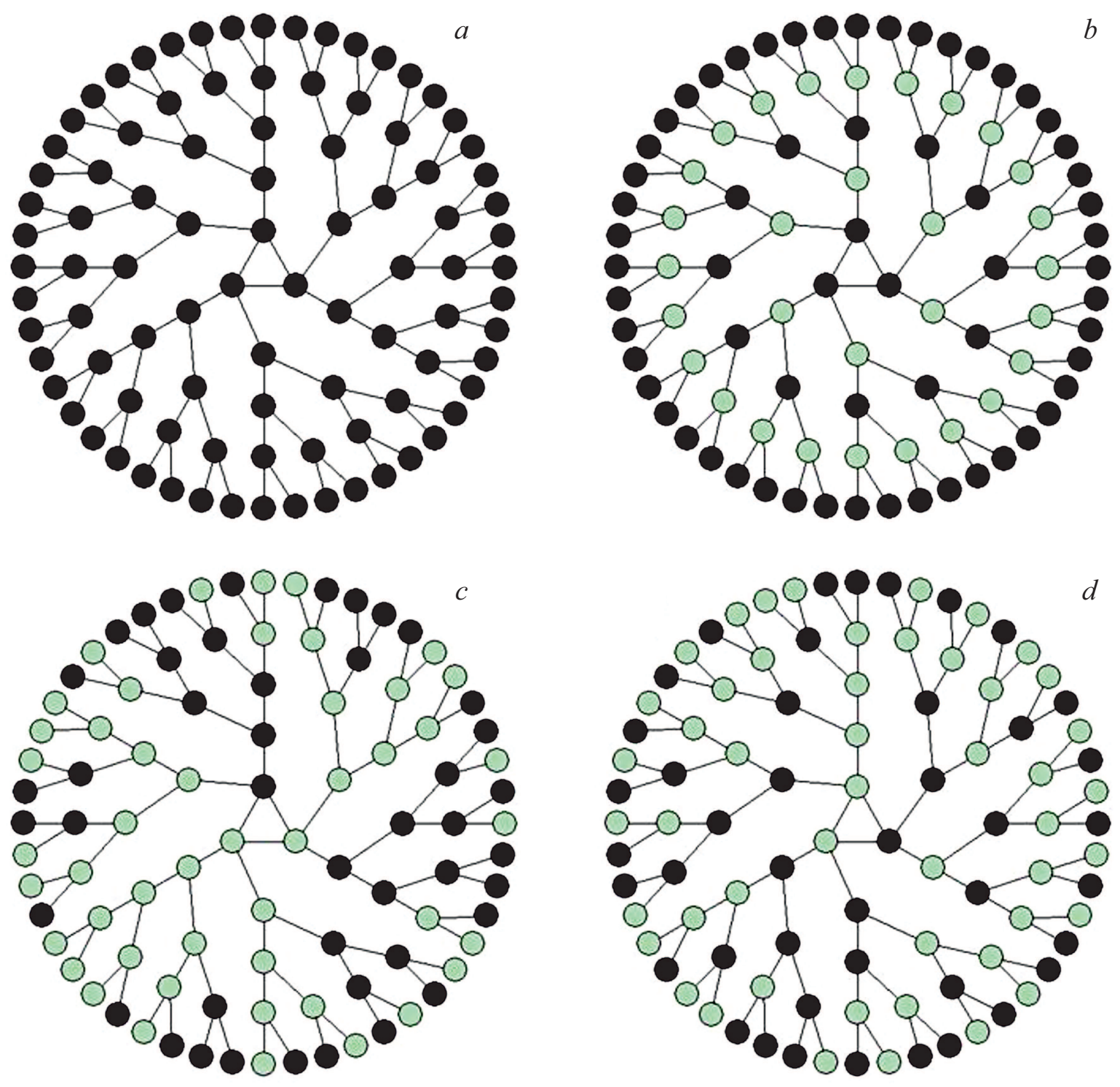

Рис. 3. Структуры основного состояния для дендримера радиуса $R=6$ при: $a-J_{1}=1, J_{2}=1 ; b-J_{1}=-1, J_{2}=1 ; c-J_{1}=1$, $J_{2}=-1 ; d-J_{1}=-1, J_{2}=-1$.

при достижении энергетического минимума нами проводился анализ магнитной структуры основного состояния. При этом проводилось сравнение данной конфигурации с полученными ранее, и при обнаружении новой уникальной конфигурации производится ее сохранение в специальной базе данных и вывод в текстовый файл всей необходимой информации (координаты каждого спина, его значение, внутренняя энергия системы и т.д.) а также запись в графический файл. После акта сохранения конфигурации следующая процедура проводится только после достижения системой энергетического максимума и повторного возвращения к минимуму. Данная процедура позволяет избежать дублирования многократно встречающихся состояний с одинаковой магнитной структурой. Таким образом, если основное состояние не вырождено, то в базе данных в конце процесса моделирования окажется некоторое ограниченное количество конфигураций (для ферромагнитной модели Изинга, к примеру, с учетом симметрии относительно одновременного отражения всех спинов на решетке в базе окажется 2 магнитные конфигурации), Для фрустрированных систем количество таких конфигураций будет бесконечным, при этом для экономии дискового пространства в памяти сохраняется не более 100 конфигураций. Более подробно алгоритм Ванга-Ландау изложен в работах [8-18].

Рассчитанная таким образом плотность состояний $g(E)$ для систем с различными размерами $R$ при $J_{1}=-1$ и $J_{2}=1$ приведена на рис. 2 (здесь и далее статистическая погрешность не превышает размеров символов, использованных для построения зависимостей). Расчеты проводились для дендримеров с числом генераций $R=2-6$. Для удобства восприятия в виде символов 
приведены лишь некоторые данные, линия отображает все точки. Из графика видно, что вырождение основного состояния в данном случае отсутствует.

Таким образом, вычислив плотность состояний системы можно достаточно легко рассчитать значения любых термодинамических параметров при любой интересующей нас температуре. В частности, внутреннюю энергию $U$, свободную энергию $F$, энтропию $S$, теплоемкость $C$, намагниченность $m$ можно вычислить, используя следующие выражения:

$$
\begin{gathered}
U(T)=\frac{\sum_{E} E g(E) e^{-E / k_{\mathrm{B}} T}}{\sum_{E} g(E) e^{-E / k_{\mathrm{B}} T}} \equiv\langle E\rangle_{T}, \\
F(T)=-k_{\mathrm{B}} R \ln \left(\sum_{E} g(E) e^{-E / k_{\mathrm{B}} T}\right), \\
S(T)=\frac{U(T)-F(T)}{T}, \\
D C=\left(N K^{2}\right)\left(\left\langle U^{2}\right\rangle-\langle U\rangle^{2}\right) \\
m(T)=\frac{\sum_{E}\langle m\rangle_{E} g(E) e^{-E / k_{\mathrm{B}} T}}{\sum_{E} g(E) e^{-E / k_{\mathrm{B}} T}} \equiv\langle m\rangle_{T},
\end{gathered}
$$

где $K=\left|J_{1}\right| / k_{\mathrm{B}} T, N-$ число частиц.

\section{3. Результаты моделирования}

На рис. 3 приведены магнитные структуры основного состояния для исследуемой модели при различных значениях обменных взаимодействий $J_{1}$

Черными кружками изображены спины направленные вверх, а светлыми - вниз. Вставка а соответствует случаю $J_{1}=1, J=1$, основное состояние при этом ферромагнитное. При $J_{1}=-1, J_{2}=1$ в системе реализуется антиферромагнитная структура, при котором спины ближайших генераций ориентируются ан-

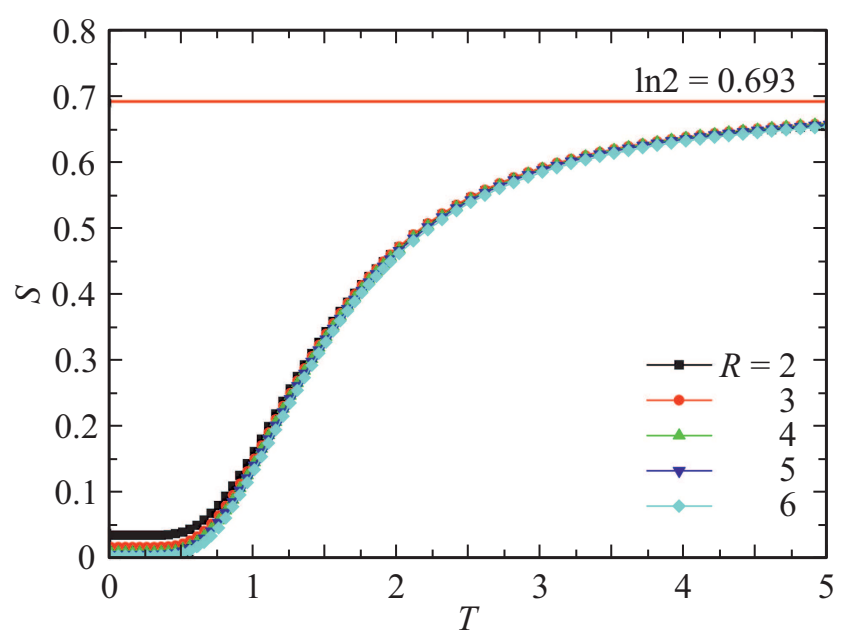

Рис. 4. Температурная зависимость энтропии $S$ для систем с размерами $R$ при $J_{1}=-1$ и $J_{2}=1$.

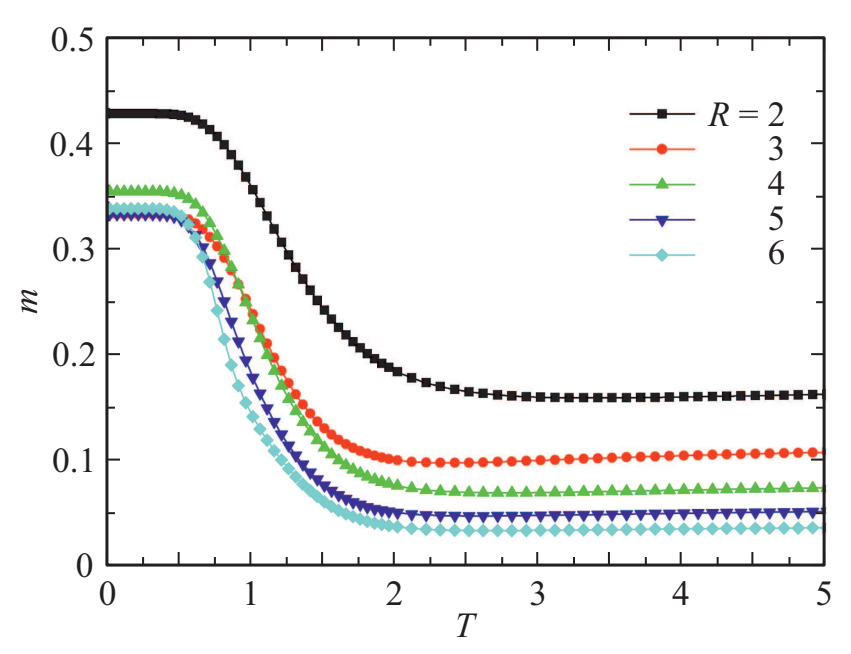

Рис. 5. Температурная зависимость намагниченности $m$ для систем с размерами $R$ при $J_{1}=-1$ и $J_{2}=1$.

типараллельно. При этом следует отметить, что количество узлов в подрешетках различается, в следствие чего система также обладает ненулевым магнитным моментом. В случае антиферромагнитного взаимодействия между соседними узлами одной генерации $\left(J_{2}=-1\right)$, как показано на рис. $3, c$ и $d$, система становится сильно вырожденной, упорядочение в системе не возникает и система становится сильно фрустрированной.

Далее мы приводим результаты расчета температурных зависимостей различных термодинамических параметров при $J_{1}=-1, J_{2}=1$, рассчитанные из приведенной на рис. 2 плотности состояний $g(E)$.

Температурные зависимости энтропии $S$ при различных размерах системы $R$ приведена на рис. 4 (здесь и далее температура дана в единицах $\left.\left|J_{1}\right| / k_{\mathrm{B}}\right)$. С увеличением температуры энтропия системы стремится к теоретически предсказанному значению $\ln 2$. При низких температурах, близких к абсолютному нулю, энтропия системы стремится к нулю.

Температурные зависимости намагниченности $m$ при различных размерах системы приведены на рис. 5. Как видно из рисунка, при низких температурах система стремится к состоянию с остаточной намагниченностью $m=1 / 3$, так как число спинов в четной подрешетке, направленных вверх примерно два раза больше числа спинов в нечетной подрешетке. Также отличительной особенностью являются высокотемпературные хвосты намагниченности, сохраняющиеся даже для систем больших размеров. Доля поверхностных узлов в дендримерах больших размеров близка к 50\%, в то время как для систем на обычных решетках стремится к нулю.

На рис. 6 и 7 представлены температурные зависимости внутренней энергии $E$ и теплоемкости $C$. Отметим слабо выраженный максимум теплоемкости, также обусловленный большим числом поверхностных узлов. 


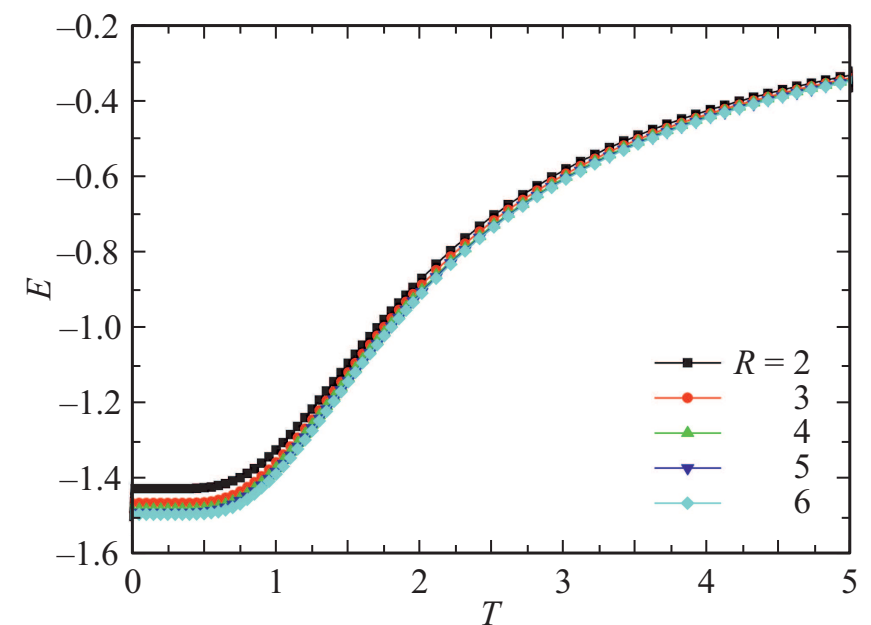

Рис. 6. Температурная зависимость внутренней энергии $E$ для систем с размерами $R$ при $J_{1}=-1$ и $J_{2}=1$.

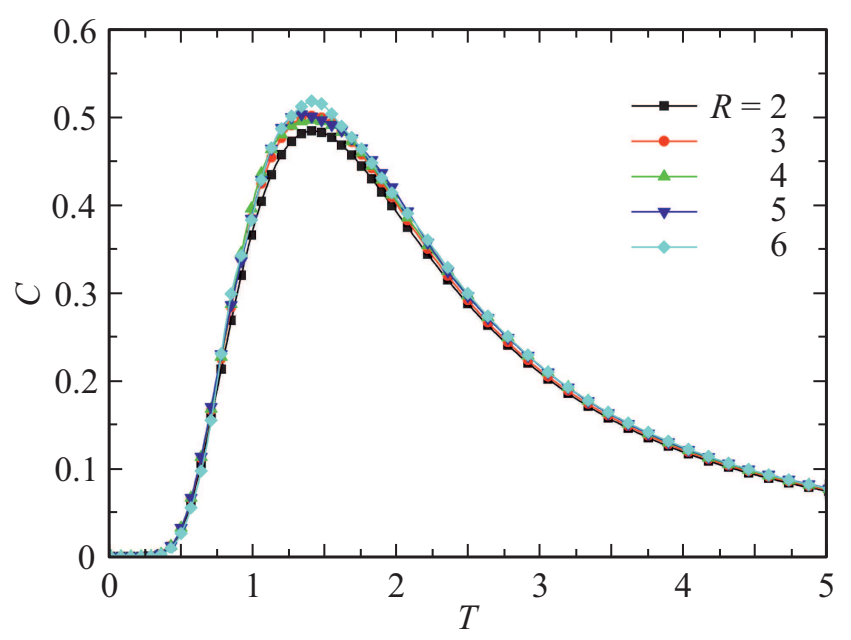

Рис. 7. Температурная зависимость теплоемкости $C$ для систем с размерами $R$ при $J_{1}=-1$ и $J_{2}=1$.

Фазовый переход из упорядоченного ферримагнитного состояния в парамагнитное состояние происходит при критической температуре $T_{c} \approx 1.5$.

\section{4. Заключение}

Таким образом, в данной работе проведены исследования моделей магнитных дендримеров с использованием высокоэффективного алгоритма Ванга-Ландау метода Монте-Карло. Проведен анализ структур основного состояния при различных значениях обменных взаимодействий. Показано, что в зависимости от величин обменных взаимодействий система может иметь ферромагнитное или ферримагнитное упорядочение, а также быть сильно вырожденной и неупорядоченной - в системе возникает фрустрация. Рассчитаны температурные зависимости различных термодинамических параметров системы. Показано, что в модели дендримера число поверхностных узлов близко к половине от общего числа узлов, в следствие чего с ростом размеров системы влияние поверхностных узлов на общую картину поведения системы не ослабевает.

\section{Финансирование работы}

Исследование выполнено при финансовой поддержке Российского фонда фундаментальных исследований в рамках проекта 18-32-20098-мол-а-вед.

\section{Конфоликт интересов}

Авторы заявляют, что у них нет конфликта интересов.

\section{Список литературы}

[1] V. Biricova, A. Laznickova. Bioorg. Chem. 37, 185 (2009).

[2] U. Boas, P.M. Heegaard. Chem. Soc. Rev. 33, 43 (2004).

[3] B. Pan, F. Gao, L. Ao. J. Magn. Magn. Mater. 293, 252 (2005).

[4] M.A. Mintzer, M.W. Grinstaff. Chem. Soc. Rev. 40, 173 (2011).

[5] J.S. Klos, J.U. Sommer. Soft Matter. 12, 9007 (2016).

[6] A. Jabar, R. Masrour. Phase Trans. 90, 1112 (2017).

[7] C.C. Lee, J.A. MacKay, J.M.J. Fréchet, F.C. Szoka. Nature Biotechnol. 23, 1517 (2005).

[8] М.А. Магомедов, А.К. Муртазаев, Л.К. Магомедова. Вестн. ДГУ 31, 71 (2016).

[9] A.K. Murtazaev, A.B. Babaev, M.A. Magomedov, F.A. Kassan-Ogly, A.I. Proshkin. Solid State Commun. 246, 41 (2016).

[10] М.А. Магомедов, А.К. Муртазаев, Л.К. Магомедова. Вестн. ДГУ 31, 43 (2016).

[11] М.А. Магомедов, А.К. Муртазаев. ФТТ 60, 1173 (2018).

[12] F. Wang, D.P. Landau. Phys. Rev. E 64, 056101 (2001).

[13] D.P. Landau, S.-H. Tsai, M. Exler. Am. J. Phys. 72, 1294 (2004).

[14] C.J. Silva, A.A. Caparica, J.A. Plascak. Phys. Rev. E 73, 36702 (2006).

[15] M.K. Ramazanov, A.K. Murtazaev, M.A. Magomedov. Solid State Commun. 233, 35 (2016).

[16] M.K. Ramazanov, A.K. Murtazaev, M.A. Magomedov. Physica A 521, 543 (2019).

[17] А.К. Муртазаев, М.К. Рамазанов, М.К. Мазагаева, М.А. Магомедов. ЖЭТФ 156, 502 (2019).

[18] A.G. Cunha-Netto, A.A. Caparica, S.H. Tsai, R. Dickman, D.P. Landau. Phys. Rev. E 78, 55701 (2008).

Редактор Д.В. Жуманов 